\title{
Mouse Adipose-Derived Stem Cells Undergo Multilineage Differentiation in Vitro but Primarily Osteogenic and Chondrogenic Differentiation in Vivo
}

\author{
BO ZHENG, M.D., BAOHONG CAO, Ph.D., M.D., \\ GUANGHENG LI, Ph.D., M.D., and JOHNNY HUARD, Ph.D.
}

\begin{abstract}
Human, rat, and mouse studies have demonstrated the existence of a population of adipose-derived adult stem (ADAS) cells that can undergo multilineage differentiation in vitro. However, it remains unclear whether these cells maintain their multilineage potential in vivo. The aim of this study was to examine the in vitro and in vivo characteristics and behavior of a potential population of murine ADAS (muADAS) cells isolated from the visceral fat of the abdominal cavity of $\mathrm{C57BL} / 10 \mathrm{~J}$ mice. We used flow cytometry to examine the cells' expression of CD29, CD31, CD45, CD34, CD44, CD144, CD146, Flk1, and Sca-1. The isolated cell population was CD45 negative, which precludes contamination by hematopoietic cells, but was partially positive for Sca-1 and CD34: 2 stem-cell markers. After induction in conditioned medium, the muADAS cells gained the ability to undergo adipogenic, osteogenic, chondrogenic, myogenic, and hematopoietic differentiation in vitro. The muADAS cells readily differentiated to form bone and cartilage in vivo for up to 24 weeks, but their ability to regenerate muscle or reconstitute bone marrow was found to be limited.
\end{abstract}

\section{INTRODUCTION}

$\mathbf{R}$ ESEARCHERS TRADITIONALLY HAVE BELIEVED that stem cells in adult tissues can generate only the types of cells present within the tissues in which the stem cells reside; however, increasing numbers of papers have reported the detection of pluripotent stem and progenitor cells in various types of adult tissues, including bone marrow, muscle, brain, skin, and adipose tissue. ${ }^{1-6}$ These cells, which purportedly can differentiate toward various lineages both in vitro and in vivo, could serve as the basis for a wide variety of tissue regeneration and repair applications. Unfortunately, the isolation of pluripotent stem cells from most of the tissue sources investigated has practical limitations. Often, the source tissue is not abundant, or cell harvesting relies on an invasive technique (e.g., bone marrow isolation) or is clinically impossible (e.g., brain tissue isolation). Ideal stem cell sources would be easily accessible and available in unlimited amounts. Because adipose tissue fits these criteria, it is an attractive candidate as a stem cell source. Scientists recently have demonstrated that certain cells isolated from subcutaneous or visceral fat tissue can differentiate into a variety of cell types in vitro, including adipocytes, osteoblasts, chondrocytes, and myoblasts. ${ }^{6-8}$ These cells have been termed "adipose-derived adult stem (ADAS) cells" because they are self-renewing and can be induced to differentiate toward various mesodermal lineages. ${ }^{9,10}$

Adipose tissue, which is abundant, expendable, and easy to obtain from the body, is derived from the mesoderm and contains 2 different cell populations: mature adipose cells and stroma vascular fraction (SVF) cells. ${ }^{11} \mathrm{SVF}$ cells isolated from rat adipose tissue have exhibited multipotent differentiation in vitro. ${ }^{10,12}$ The aim of our study was to determine whether visceral adipose tissue from mice contains

Growth and Development Laboratory, Children's Hospital of Pittsburgh and Department of Orthopaedic Surgery, University of Pittsburgh, Pittsburgh, Pennsylvania. 
a population of adipose-derived mesodermal stem cells that can undergo multilineage differentiation in vitro and in vivo. The existence of such stem cells would validate the notion that these cells reside in adipose tissue and can be used to improve the healing of various types of tissues.

\section{MATERIALS AND METHODS}

\section{Animals}

The normal mice (C57BL/10J and BALB/cJ), $m d x$ mice (C57BL/10ScSn DMD ${ }^{\mathrm{mdx}} / \mathrm{J}$ ), SJL/J mice, and SCID mice used in this study were purchased from The Jackson Laboratory (Bar Harbor, ME). The Children's Hospital of Pittsburgh's Institutional Animal Care and Use Committee approved all animal protocols used for these experiments.

\section{Cell harvest and culture}

For isolation of murine adipose-derived adult stem (muADAS) cells, visceral adipose tissue was excised separately from six $\mathrm{C} 57 \mathrm{BL} / 10 \mathrm{~J}$ mice and five $\mathrm{BALB} / \mathrm{cJ}$ mice (4-8 weeks of age), finely minced, and digested using $0.075 \%$ collagenase type XI (Sigma, St. Louis, MO) for $45 \mathrm{~min}$ at $37^{\circ} \mathrm{C}$. Enzyme activity was neutralized by treatment with Dulbecco's modified Eagle's medium (DMEM) containing $10 \%$ fetal bovine serum (FBS). The cell suspension was centrifuged at $1200 \mathrm{~g}$ for $10 \mathrm{~min}$ to separate the floating adipocytes from the SVF. The SVF was resuspended in ammonium chloride $(160 \mathrm{mM})$, incubated at room temperature for $10 \mathrm{~min}$ to lyse contaminating red blood cells, and then centrifuged as described above. After filtration of the cell suspension through a $100-\mu$ m nylon strainer to remove cellular debris, cell number and viability were determined using trypan blue exclusion. The SVF cells were plated in T75 flasks overnight in control medium (DMEM, $10 \% \mathrm{FBS}, 1 \%$ penicillin/streptomycin) at $37^{\circ} \mathrm{C}$ in $5 \%$ carbon dioxide $\left(\mathrm{CO}_{2}\right)$. After incubation for $24 \mathrm{~h}$, the flasks were washed extensively uisng phosphate buffered solution (PBS) to remove residual nonadherent cells. The adherent muADAS cells were expanded using serial passaging.

\section{Characterization of muADAS cells}

Flow cytometry was used to characterize muADAS cells obtained from C57BL/10J and BALB/cJ mice and grown for 4 passages and 10 passages under control conditions. Cultured cells were trypinized, spun, and washed in cold PBS 1X (Mediatech, Herndon, VA) containing 2\% FBS. The cells then were divided into aliquots and were spun to form a pellet. Cells were blocked using mouse serum (Sigma) diluted 1:10 in PBS and rat anti-mouse CD16/CD32 (BD PharMingen, San Diego, CA) for $10 \mathrm{~min}$ on ice. The primary antibodies (applied in optimal amounts) included a biotin-conjugated rat anti-mouse monoclonal antibody against CD34 and CD29 (surface proteins used to define mesenchymal stem cells and progenitor cells) followed by streptavidin-APC, PE-conjugated mouse anti-Sca-1, and FITC-conjugated mouse anti-CD45 and anti-CD44 (all from BD PharMingen, San Diego, CA). To exclude dead cells, 7-aminoactinomycin D (Via-Probe, BD PharMingen) was added to each tube. Live cells were analyzed using a FACSCalibur flow cytometer (Becton Dickinson, San Jose, CA) and CellQuest software (Becton Dickinson). Analysis of surface protein expression was performed using appropriate gating on viable CD45-negative cells to eliminate contaminating hematopoietic cells. The isotype antibody control samples obtained for each individual cell population were used to set the dot-plot intercepts used for the analysis.

\section{In vitro adipogenic differentiation assay}

A previously described method was used to analyze adipogenesis. ${ }^{6}$ Trypsin/ethylenediaminetetraacetic acid was used to harvest the muADAS cells, which then were replated in 6-well plates $(100,000$ cells per well) in control medium to allow attachment. Twenty-four $\mathrm{h}$ later, the medium was replaced with new control medium or adipose medium (control medium plus insulin $[10 \mu \mathrm{M}]$, dexamethasone $[1 \mu \mathrm{M}]$, isobutyl-methylxanthine $[0.5 \mathrm{mM}]$, and indomethacin [200 $\mu \mathrm{M}$ ] [all from Sigma-Aldrich]). Cultures were maintained for 14 days, and medium was changed every 2 days. The cultures then were assessed using Oil Red O stain, which serves as an indicator of intracellular lipid accumulation. The cells were fixed for $10 \mathrm{~min}$ at room temperature in $10 \%$ neutral buffered formalin and were washed with distilled water. They then were incubated in Oil Red $\mathrm{O}$ reagent for $30 \mathrm{~min}$ and washed 3 times with distilled water. The cells were counterstained with hematoxylin for $1 \mathrm{~min}$.

\section{In vitro chondrogenic differentiation assay}

Pellet culturing was performed as described previously. ${ }^{13-15}$ Cells $(n=250,000)$ were placed in a $15-\mathrm{mL}$ conical polypropylene tube and were centrifuged at $600 \mathrm{~g}$ for $5 \mathrm{~min}$; they then were left on the bottom of the tube and cultured in control medium or defined medium: highglucose DMEM supplemented with 1\% ITS+Premix (BD Biosciences, Bedford, MA), L-ascorbic acid-2-phosphate (0.1 mM, Wako Pure Chemical Industries, Ltd. Osaka, Japan $)$, dexamethasone $\left(1 \times 10^{-7} \mathrm{M}\right.$, Sigma $)$, proline $(400 \mu \mathrm{g} /$ $\mathrm{mL}$, Sigma), and bone morphogenetic protein 4 (BMP4) (500 ng/mL, R\&D Systems). After the addition of $1 \mathrm{~mL}$ of chondrogenic medium to each tube, the pelleted cells were incubated at $37^{\circ} \mathrm{C}$ in $5 \% \mathrm{CO}_{2}$. The medium was changed every 2 to 3 days. Pellets were harvested at different time points (Days 7, 14, 21) and were embedded in paraffin as detailed in the standard protocol (available on the Cambrex Company website). Chondrogenesis was confirmed by use of the histologic stain Alcian blue at a low $\mathrm{pH}$ to stain the highly sulfated proteoglycans that are characteristic of cartilaginous matrix. After that, sections were counterstained with nuclear fast red, which specifically stains cell nuclei. 


\section{In vitro osteogenic differentiation assay}

The in vitro osteogenic assay was performed as described previously. ${ }^{6}$ Osteogenic differentiation was induced by culturing muADAS cells in osteogenic medium (OM, control medium supplemented with dexamethasone $[0.1 \mu \mathrm{M}]$, ascorbate-2-phosphate $[50 \mu \mathrm{M}]$, and $\beta$-glycerophosphate $[10 \mathrm{mM}$ ] [all from Sigma]). The medium was changed every 3 days. Osteogenesis was assessed using von Kossa staining and observation of alkaline phosphatase (ALP) activity 2 and 4 weeks after initial osteogenic induction, as described previously. ${ }^{16}$ The cells were incubated in OM for 2 weeks and rinsed with PBS. The commercially available Sigma AP kit 86-c (Sigma) then was used to detect ALP activity. The cells used for von Kossa staining were incubated in OM for 4 weeks and fixed at room temperature with $4 \%$ paraformaldehyde for $1 \mathrm{~h}$. They were rinsed with distilled water and then were incubated for $30 \mathrm{~min}$ in $1 \%$ silver nitrate solution in the dark. The cells then were rinsed again with distilled water and exposed to ultraviolet light for $60 \mathrm{~min}$. Secreted calcified extracellular matrix appeared as black nodules after von Kossa staining.

\section{In vivo chondrogenic and osteogenic differentiation assays}

muADAS cells were seeded at a density of $1.5 \times 10^{6}$ cells in $75-\mathrm{cm}^{2}$ flasks and were grown to $50 \%$ confluence. On the day of transduction, cells were washed twice with sterile PBS and were incubated for $16 \mathrm{~h}$ with a mixture of $2 \mathrm{~mL}$ of retro-LacZ viral suspension $\left(1 \times 10^{6}-5 \times 10^{6} \mathrm{cfu} / \mathrm{mL}\right)$, $18 \mathrm{~mL}$ of DMEM supplemented with $10 \% \mathrm{FBS}$, and polybrene $(8 \mu \mathrm{g} / \mathrm{mL})$ in $5 \% \mathrm{CO}_{2}$ at $37^{\circ} \mathrm{C}$. This same solution was replaced every $16 \mathrm{~h}$ for $32 \mathrm{~h}$. The percentage of LacZ-positive transduced cells (muADAS-LacZ cells) was determined using X-galactosidase (gal) staining. The aforementioned technique also was used to transduce muADAS-LacZ cells with a retro- $B M P 4$ virus. Medium containing $10 \mathrm{~mL}$ of retro-BMP4 viral suspension $\left(5 \times 10^{5} \mathrm{cfu} / \mathrm{mL}\right), 10 \mathrm{~mL}$ of DMEM with $10 \% \mathrm{FBS}$, and polybrene $(8 \mu \mathrm{g} / \mathrm{mL})$ was added to the flasks. muADAS-LacZ cells were cultured in this medium for $48 \mathrm{~h}$, with medium changes performed every 16h. After transduction, the muADAS-LacZ-BMP4 cells were cultured in 6-well plates until the cultures reached confluence. The culture medium was collected after $48 \mathrm{~h}$, centrifuged at $2000 \mathrm{rpm}$ and $4^{\circ} \mathrm{C}$ for $5 \mathrm{~min}$ to remove cellular debris, and then used to perform a $B M P 4$ bioassay. A previously described $B M P 4$ assay was used to determine the level of functional $B M P 4$ secreted by the transduced cells. ${ }^{17}$ After detection of bioactive $B M P 4$ in the culture medium $(100 \pm 22 \mathrm{ng} / \mathrm{million}$ cells/24 h) of muADAS-LacZ-BMP4 cells, $3 \times 10^{5}$ cells in a $100-\mu \mathrm{L}$ cell suspension were seeded on the surface of a $6 \mathrm{~mm} \times 6 \mathrm{~mm}$ piece of sterile collagen sponge (Gelfoam, Pharmacia \& Upjohn Co., Ann Arbor, MI). After the Gelfoam absorbed all the cell suspension, $3 \mathrm{~mL}$ of DMEM supplemented with $10 \%$ FBS was added to each well; the wells then were placed in a cell incubator overnight.

Animal surgery was performed on the following day. Samples were implanted into the skeletal muscle pouch of the gluteofemoral muscle of each SCID mouse. The mice were examined radiographically and then killed at different times $(2,4,5$, and 24 weeks) after cell implantation. The harvested tissue samples obtained 2, 4, and 5 weeks after cell implantation were treated with CRYO-GEL Embedding Medium (Cancer Diagnostics, Inc., Birmingham, MI), rapidly frozen, and stored at $-80^{\circ} \mathrm{C}$. Frozen sections were fixed in $1 \%$ glutaraldehyde for $1 \mathrm{~min}$ and then were washed 3 times in PBS. Some of the sections were stained in X-gal solution and counterstained with eosin. Other sections were stained in X-gal solution and immunostained with collagen type II (1:200 dilution, Santa Cruz Biotech, Santa Cruz, CA) and osteocalcin (1:200 dilution, Santa Cruz Biotech), as detailed in the manufacturer's protocol (Vectastain Elite ABC kit, Vector Laboratories, Burlingame, CA). Some of the 24-week samples were frozen and sectioned, whereas others were decalcified and embedded in paraffin. The frozen sections were stained in X-gal solution and counterstained with eosin. The paraffin-embedded sections were stained with Alcian blue and eosin. Some sections were immunostained for collagen type II or osteocalcin in addition to $\beta$-gal (1:200 dilution, Abcam, Inc., Cambridge, UK).

In vitro and in vivo myogenic differentiation assays

Myogenic differentiation was induced by culturing muADAS cells in myogenic medium (MM: control medium supplemented with dexamethasone [0.1 $\mu \mathrm{M}$, Sigma], hydrocortisone [50 $\mu \mathrm{M}$, Sigma], and 5\% horse serum) for 4 weeks and was confirmed using desmin staining. The cells were rinsed twice with PBS, fixed for $1 \mathrm{~min}$ with methanol, and again washed several times with PBS. The cells were blocked with $5 \%$ horse serum at room temperature for $1 \mathrm{~h}$ and then were incubated with the primary antibody rabbit immunoglobulin G (IgG) anti-desmin (1:250 in PBS, Sigma) overnight at $4{ }^{\circ} \mathrm{C}$. After being rinsed thoroughly with PBS, cells were incubated with biotinylated anti-rabbit IgG antibody (1:250, Sigma) for $1 \mathrm{~h}$ at room temperature. The cells then were rinsed with PBS and incubated for $30 \mathrm{~min}$ at room temperature with streptavidin conjugated $\mathrm{Cy} 3$ fluorochrome (1:300, Sigma). Slides were viewed with a Nikon Diaphot 300 fluorescence microscope (Nikon, Garden City, NY).

In vivo myogenic differentiation was evaluated by injection of muADAS cells into the skeletal muscle of $m d x$ mice, which model Duchenne muscular dystrophy. ${ }^{18}$ muADAS cells from passages 3 to 5 were grown to $60 \%$ confluence in control medium. To enable tracking of cells after injection into gastrocnemius muscles, muADAS cells were genetically engineered to express the $L a c Z$ reporter 
gene (muADAS-LacZ cells), as described above for the in vivo chondrogenic and osteogenic assays. The cells were harvested, mixed with FluoSpheres (Molecular Probes, Eugene, OR) in $20 \mu \mathrm{L}$ of Hanks' balanced salt solution (HBSS, Invitrogen, Grand Island, NY), and injected $\left(5 \times 10^{5}\right.$ cells per injection) into a single site within the gastrocnemius muscles of 8 -week-old $m d x$ mice. Injected gastrocnemius muscles were harvested on Day 15 and were stained for $L a c Z$ and dystrophin. For $L a c Z$ staining, the sections were fixed in $1 \%$ glutaraldehyde, incubated overnight with $\mathrm{X}$-gal substrate at $37^{\circ} \mathrm{C}$, and counterstained with eosin. The sections were mounted in Gel/Mount (M01, Biomeda, Plovdiv, Bulgaria) and observed with a light microscope (Nikon Eclipse E800). For dystrophin staining, slides were fixed with acetone and blocked with $5 \%$ horse serum. The primary antibody was rabbit anti-dystrophin (1:1000, gift from Dr. T. Partridge). Sections then were washed in PBS and were incubated with biotinylated antirabbit IgG (1:300, Vector) followed by streptavidin-Cy3 (1:500, Sigma). The dystrophin-positive myofibers were visualized using fluorescence microscopy.

\section{In vitro and in vivo hematopoietic} differentiation assays

In vitro differentiation of the muADAS cells toward the hematopoietic lineage was induced by culturing the cells in methylcellulose as described previously. ${ }^{19}$ muADAS cells $\left(n=1 \times 10^{5}\right)$ in $1 \mathrm{~mL}$ of Methocult GF M3434 culture media (Stem Cell Technologies, Vancouver, Canada) were plated in a 35-mm petri dish and cultured in a humidified $5 \% \mathrm{CO}_{2}$ atmosphere maintained at $37^{\circ} \mathrm{C}$. Colonies were counted 10 days after plating. Colonies from triplicate cultures were individually lifted from the methylcellulose culture and were centrifuged to glass slides with a Cytospin (Shandon Southern, Sewickley, PA). Cells then were stained using the HEMA 3 Stain Set (Biochemical Science, Inc., Swedesboro, NJ).

In vivo hematopoietic differentiation of the muADAS cells was determined by transplantation of muADAS cells into 8- to 10 -week-old SJL/J mice that had been subjected to a single lethal dose of radiation $(10 \mathrm{~Gy}) 1$ day before cell transplantation. We used this alternative strain of mouse to distinguish the differentiated donor cells (CD45.2 positive) from the SJL/J host cells (CD45.2 negative), as described previously. ${ }^{19}$ Cells $\left(1 \times 10^{5}, 5 \times 10^{5}\right.$, or $1 \times 10^{6}$ in $400 \mu \mathrm{L}$ of HBSS) were injected intraperitoneally or intravenously into the tail vein. Mice were given acidified water and autoclaved food.

\section{RESULTS}

\section{Phenotypic characterization of the muADAS cells}

The visceral adipose tissue from 1 mouse yielded approximately $7.0 \times 10^{6}$ nucleated cells. Within 4 passages after the initial plating of the primary culture, muADAS cells appeared as a monolayer of large, flat cells. As the cells approached confluence, they acquired a spindle-shaped or fibroblast-like appearance. We used flow cytometry to evaluate the expression of CD45, Sca-1, CD34, CD44, and CD29 cell-surface antigens on the muADAS cells obtained from $\mathrm{C} 57 \mathrm{BL} / 10 \mathrm{~J}$ and $\mathrm{BALB} / \mathrm{cJ}$ mice. The muADAS cells from both mouse strains were negative for CD45, a hematopoietic cell surface marker (Fig. 1A). Approximately $31 \%$ and $10 \%$ of the muADAS cells were Sca-1 and CD34 positive, respectively (Fig. 1B). muADAS cells were negative for CD31, CD144, CD146, and flk-1 at the time of flow cytometry analysis (after 4 passages; data not shown). Because we observed similar results in cells obtained from both strains (Fig. 1C), we used only C57BL/10J mice for the remainder of the study. Flow cytometry analysis revealed that the muADAS cell marker profile changed during expansion. After passage 10, the cells showed increased Sca-1 expression (74.94\%) and decreased CD34 expression (3.25\%) (Fig. 1D).

\section{muADAS cells undergo adipogenic differentiation in vitro}

To determine whether muADAS cells undergo adipogenesis, we cultured muADAS cells in adipogenic medium. Oil Red O staining performed 2 weeks later revealed cytoplasmic lipid droplets, which are indicative of the adipogenic phenotype. These results indicate that the muADAS cells cultured in adipogenic medium underwent adipogenic differentiation (Fig. 2A), whereas muADAS cells maintained in control medium did not (Fig. 2B).

\section{muADAS cells undergo chondrogenic differentiation in vitro}

In the chondrogenic assay, the seeded muADAS cells initially formed a flat disk of cells that gradually expanded into a rounded pellet. After 21 days in culture, sections of pellet showed early cartilage formation, as indicated by Alcian blue staining $(\mathrm{pH} \mathrm{1)}$, which is specific for cartilaginous matrix (Fig. 2C). In contrast, cells cultured in control medium resulted in only light Alcian blue staining (Fig. 2D).

\section{muADAS cells undergo osteogenic differentiation in vitro}

muADAS cells cultured in osteogenic medium for 14 days expressed endogenous ALP (Fig. 2E). Moreover, mineralized nodular structures formed within 4 weeks, as confirmed by von Kossa staining (Fig. 2G). In contrast, muADAS cells cultured in control medium showed no evidence of ALP expression or formation of calcified extracellular matrix (Fig. 2F, H). 


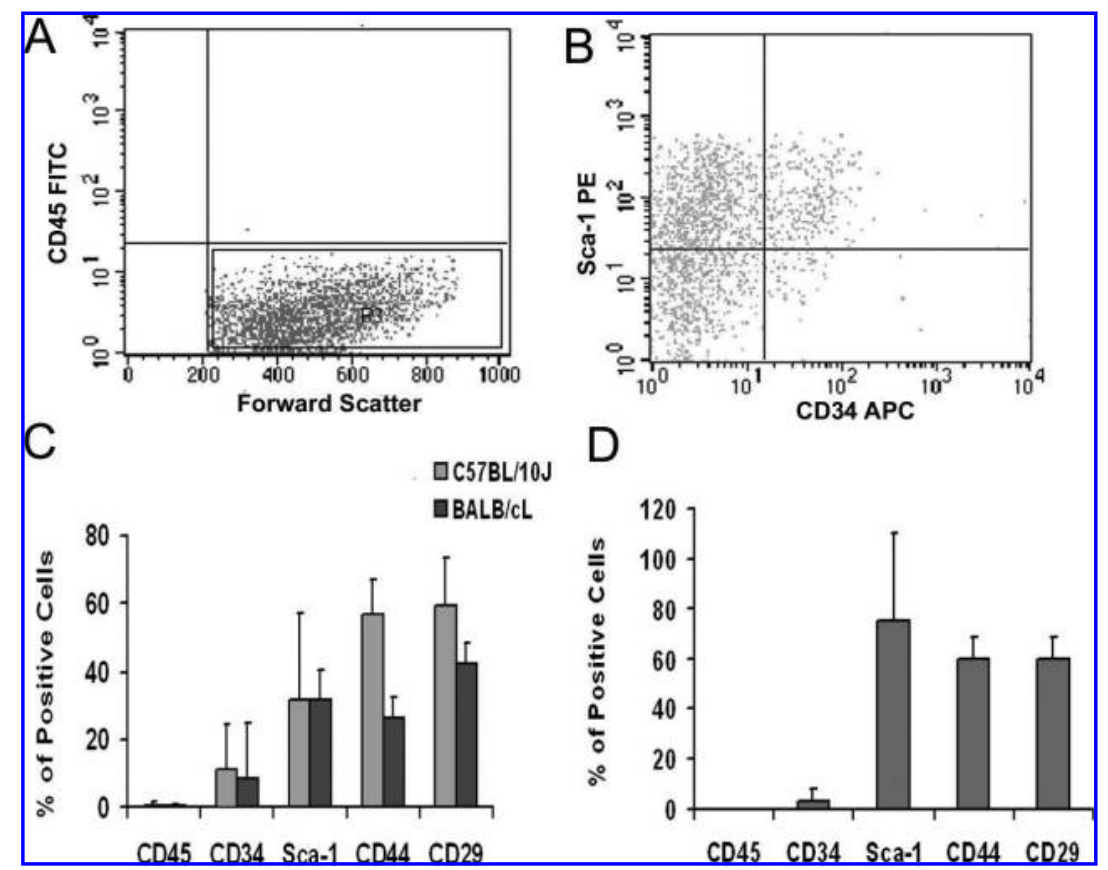

FIG. 1. (A) Flow cytometric analysis of CD45, (B) Sca-1, and CD34 expression on murine adipose-derived adult stem (muADAS) cells at passage 4. muADAS cells were CD45 negative, but $31 \%$ of them were Sca-1 positive and $10 \%$ were CD34 positive. (C) The marker profiles of muADAS cells from both C57BL/10J and BALB/cJ mice were similar. (D) CD34 expression was lower whereas Sca-1 expression was higher on C57BL/10J cells at passage 10.

\section{muADAS cells undergo chondrogenic} and osteogenic differentiation in vivo

We transduced the muADAS cells with a retrovirus containing the $L a c Z$ gene, a reporter gene that we used to track the fate of our donor cells in vivo. The LacZ gene transduction efficiency was $61 \%$. X-gal staining enabled us to track the muADAS-LacZ cells on the basis of their $\beta$-gal expression (blue nuclei) (Fig. 3A). We then cotransduced these cells with a retrovirus containing the $B M P 4$ gene. $^{17}$ The cotransduced muADAS cells secreted $100 \pm 22 \mathrm{ng}$ of $B M P 4 /$ million cells $/ 24 \mathrm{~h}$. Twenty-eight days after implantation of $3 \times 10^{5}$ muADAS cells cotransduced with $L a c Z$ and BMP4 into the host mice, bone formation was visible using radiographic examination (Fig. 3B). Histologic analysis showed that the muADAS cells differentiated toward chondrogenic and osteogenic lineages in vivo (Fig. $3 \mathrm{C}-\mathrm{F}$ ). Radiographic analysis 24 weeks after cell implantation showed solid bone formation (Fig. 4A). Histologic results indicated that cortical-like bone tissue and cartilage formed in the area of ectopic ossification (Fig. 4B, C). Immunostaining of $\beta$-gal and collagen type II or osteocalcin supports the histologic findings that the implanted muADAS cells continued to survive and participate in the generation of ectopic bone and cartilage for up to 24 weeks (Fig. 4B-F).

\section{muADAS cells' ability to undergo myogenic} differentiation in vitro and in vivo

We examined the muADAS cells' myogenic differentiation in vitro by staining for desmin and in vivo by staining for dystrophin and evaluating muscle regeneration after implanting muADAS cells into the skeletal muscle of $m d x$ mice. muADAS cells cultured for 6 weeks in myogenic medium expressed desmin (Fig. 5A). To determine the in vivo myogenic capacity of muADAS cells, we injected $5 \times 10^{5}$ muADAS-Lac $Z$ cells into the gastrocnemius muscles of $m d x$ mice. We killed the mice 15 days after injection and harvested the injected muscles to examine $\beta$-gal and dystrophin expression. Fifteen days after injection, we detected few LacZ-positive myofibers, which resulted from the fusion of donor-derived transduced muADAS cells with host myofibers (Fig. 5B, arrow). We also detected few dystrophin-positive myofibers in the injected muscle (data not shown). Our findings suggest that muADAS cells undergo limited myogenic differentiation in vitro and in vivo.

In vitro and in vivo hematopoietic differentiation by muADAS cells

To evaluate the hematopoietic differentiation of muADAS cells in vitro, we plated them in semisolid methylcellulose medium supplemented with cytokines known to promote myeloid differentiation. Of the 6 populations of muADAS cells examined, 1 formed typical hematopoietic colonies after 10 days in culture (Fig. 5C). HEMA 3 Stain Set staining also revealed granulomonocytic colonies (Fig. 5D).

We also injected 4 populations of muADAS cells intravenously (tail vein) or intraperitoneally into lethally 


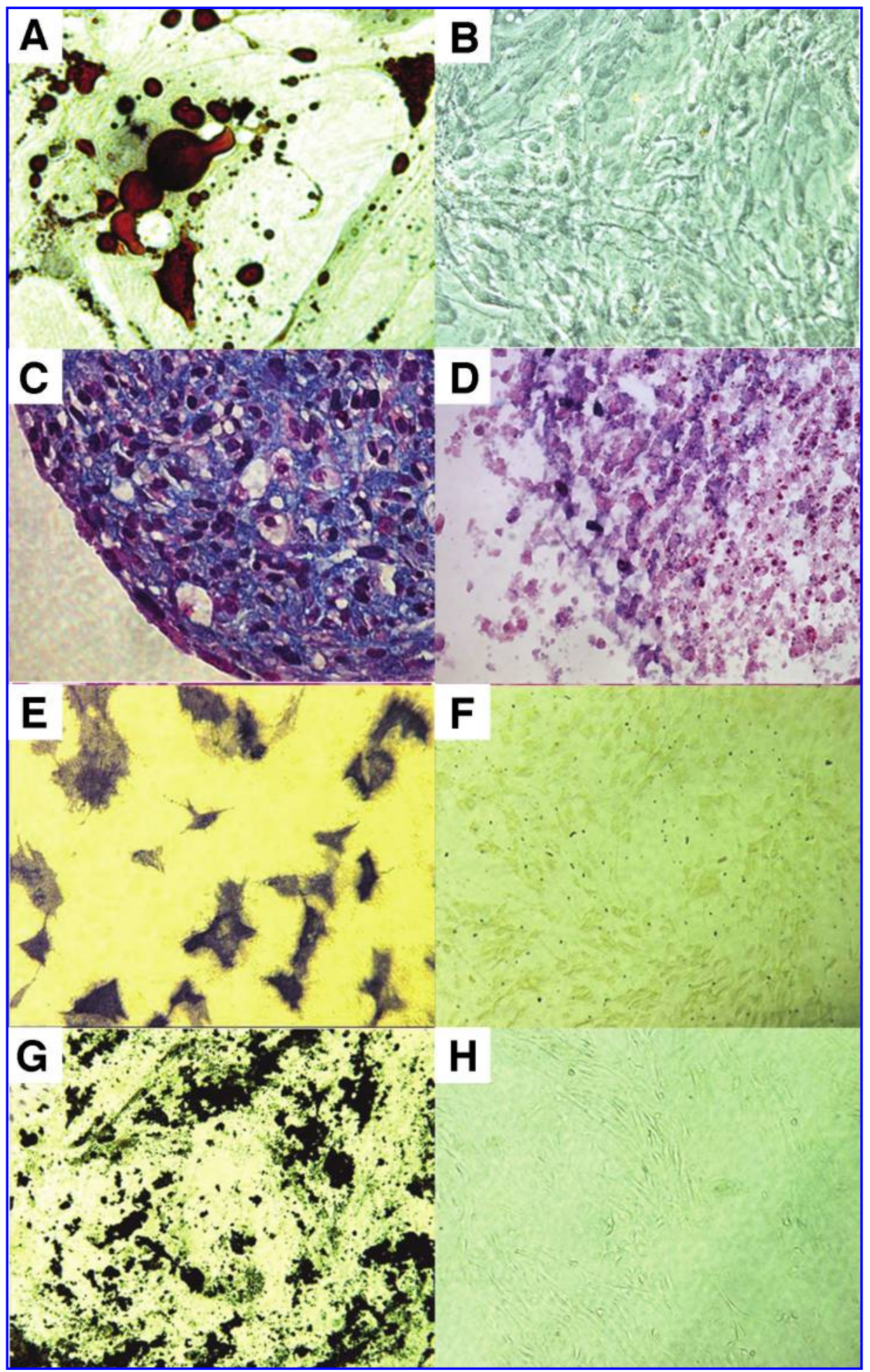

FIG. 2. Murine adipose-derived adult stem (muADAS) cells accumulated lipid-filled droplets when cultured for 2 weeks in adipogenic medium. (A) Oil Red O staining revealed lipid-filled intracellular vacuoles (magnification 40×). (B) muADAS cells maintained in control medium remained negative for Oil Red O staining (magnification 40×). (C) We observed positive Alcian blue staining, which indicates the presence of sulfated proteoglycans, in the cells cultured 3 weeks in chondrogenic medium (magnification $60 \times$ ). (D) muADAS cells maintained in control medium were only slightly positive after Alcian blue staining (magnification $60 \times$ ). (E) muADAS cells grown in osteogenic medium exhibited ALP activity (magnification 10×). (F) The cells cultured in control medium remained von Kossa negative (magnification $10 \times$ ). (G) von Kossa staining revealed calcified extracellular matrix (black) in muADAS cells cultured for 4 weeks in osteogenic medium (magnification $10 \times$ ). (H) The cells maintained in control medium remained von Kossa negative (magnification $10 \times$ ). (Color images available online at www.liebertpub.com/ten.) 


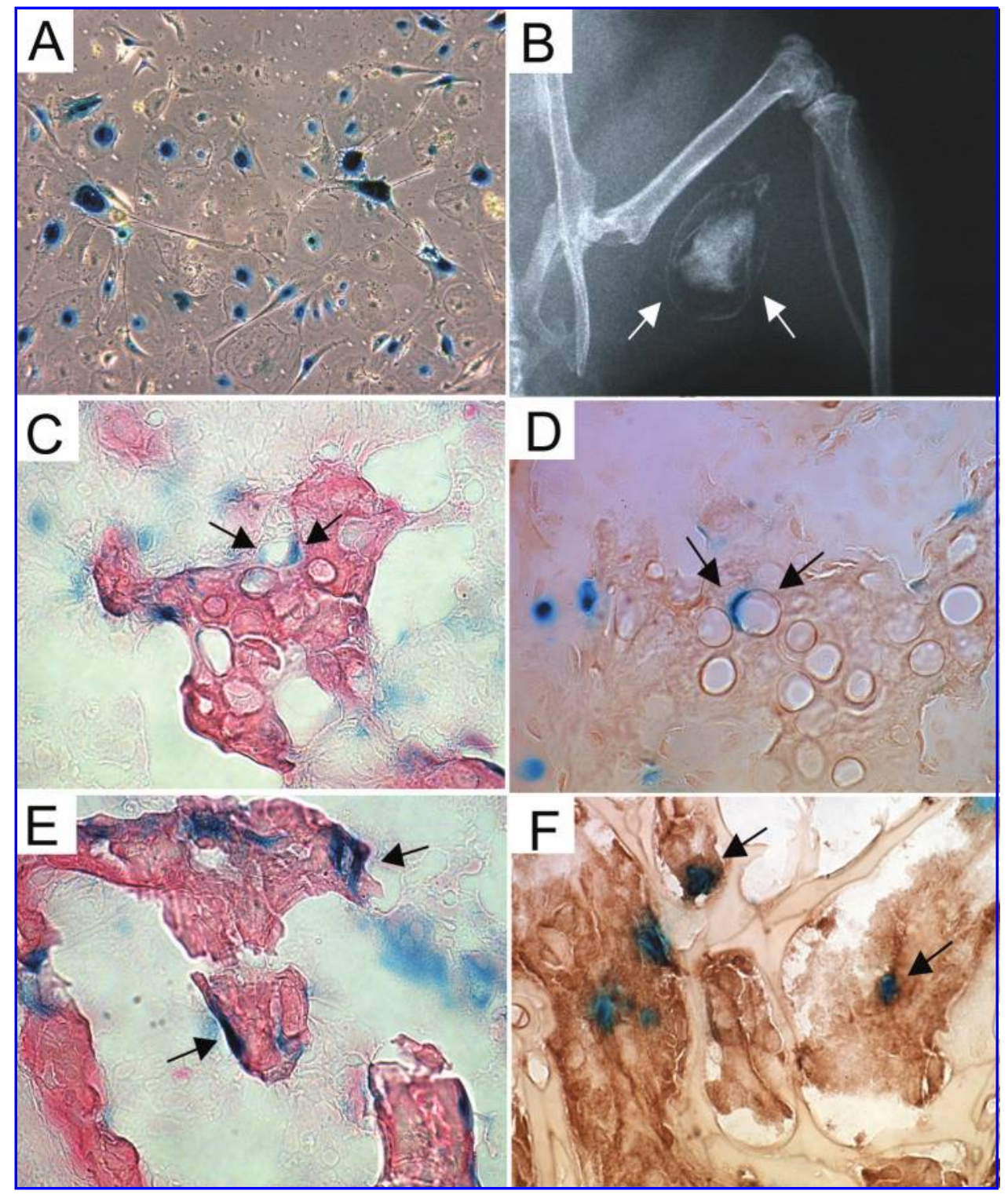

FIG. 3. (A) Murine adipose-derived adult stem (muADAS) cells were transduced with a retrovirus containing the $L a c Z$ gene and were identifiable by their LacZ-expressing nuclei after $\beta$-galactosidase staining (magnification 20×). (B) Radiographic examination showed intramuscular bone formation in SCID mice 28 days after implantation of muADAS cells cotransduced to express $L a c Z$ and $B M P 4$. (C) In vivo chondrogenesis by muADAS cells was determined by colocalization of round chondrocytes with blue nuclei (arrow) after staining for LacZ/eosin (magnification 60×) and (D) by positive immunostaining for LacZ/collagen type II (brown) (magnification 60×). (E) In vivo osteogenesis by muADAS cells was confirmed by colocalization of blue cells in the newly formed bone tissue (arrow, magnification $60 \times)($ F) with osteocalcin (brown) (arrow, magnification $60 \times$ ).

irradiated SJL/J recipient mice. The intravenous injection of $1 \times 10^{6}$ muADAS cells per mouse $(n=4$ mice $)$ induced immediate mouse death, a result that parallels findings reported by other researchers. ${ }^{11}$ After intraperitoneal transplantation of muADAS cells into 4 lethally irradiated SJL/J mice, the treated mice and control mice died within 3 weeks of transplantation. We also investigated the effect of intravenous injection (into the tail vein) of fewer cells $\left(1 \times 10^{5}\right.$ and $\left.5 \times 10^{5}\right)$ into lethally irradiated mice $(n=8$ mice, 4 per quantity of cells). The mice did not immediately die, which indicates that injection of the decreased quantities of cells did not cause fat boluses in these mice. However, the treated mice and control mice died within 3 weeks of transplantation. This finding demonstrates that the muADAS cells failed to reconstitute the bone marrow and hence failed to provide radiation protection to treated mice.

\section{DISCUSSION}

The results of this study demonstrate that a population of cells isolated from the visceral fat of mice can undergo 


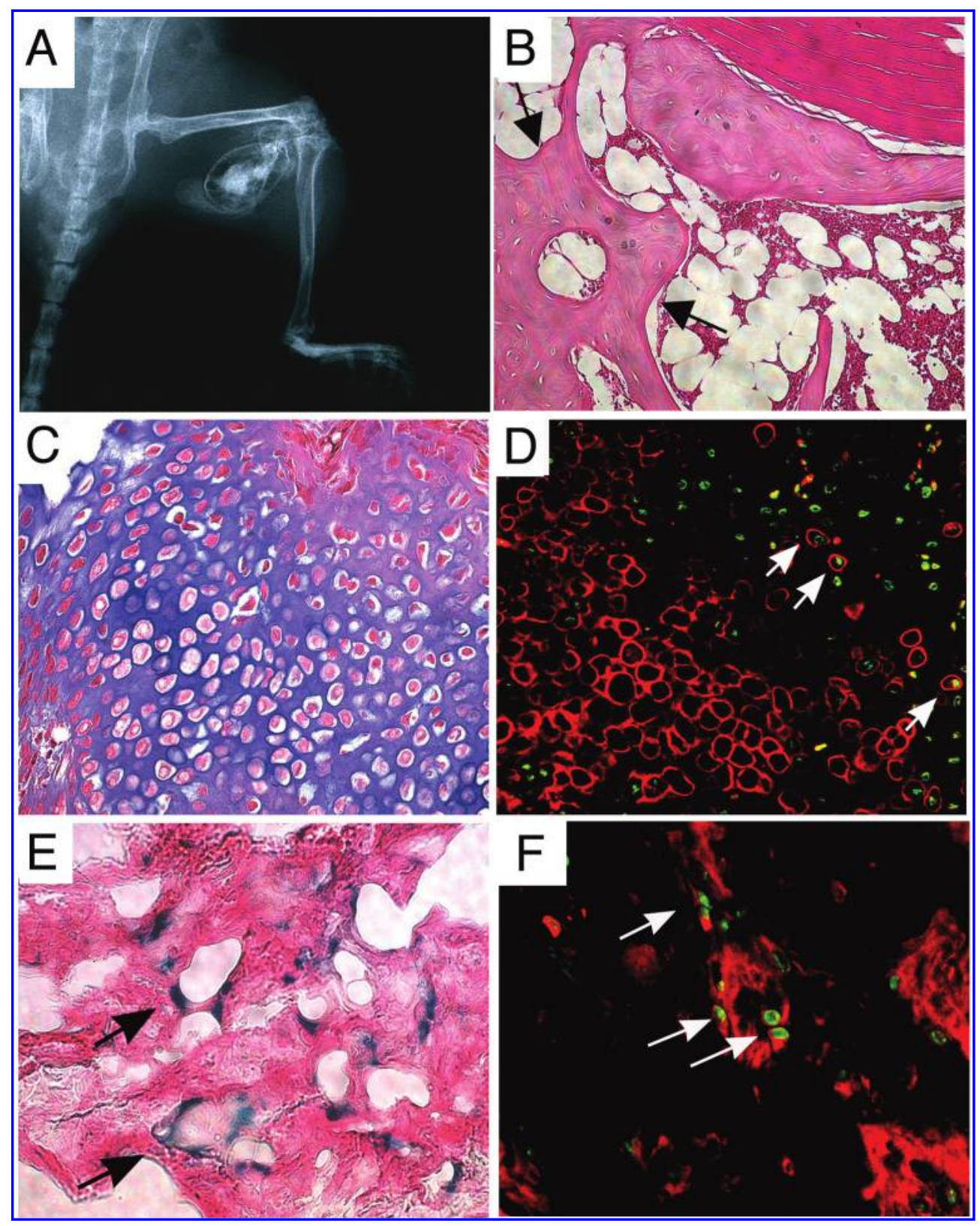

FIG. 4. Ectopic bone formation 24 weeks after implantation of muADAS-LacZ-BMP cells into muscle pockets of SCID mice. (A) Radiographic examination. (B) The histology of ectopic bone tissue (arrows, 20×). (C) In vivo ectopic bone tissue included some areas of cartilage, as shown by Alcian blue staining (magnification $40 \times$ ). (D) Chondrogenesis of murine adipose-derived adult stem (muADAS) cells was shown by positive immunostaining for colocalization of collagen type II (red)/ $\beta$-galactosidase (green) (arrow, magnification $40 \times$ ). In vivo osteogenesis by muADAS cells was confirmed (E) by LacZ/eosin (magnification $60 \times$ ) and $(\mathbf{F})$ by positive immunostaining for colocalization of osteocalcin (red)/ $\beta$-gal (green) (arrow, magnification 60x).

multilineage differentiation in vitro and in vivo. This muADAS cell population contains fibroblast-like cells that are easy to expand in vitro without specific serum supplementation. The muADAS cells used in our study differentiated toward the adipogenic, osteogenic, chondrogenic, and myogenic lineages when cultured in the presence of established lineage-specific differentiation factors. The ability of muADAS cells to undergo multilineage differentiation suggests that a stem cell population exists within mouse adipose tissue. The observed multilineage differentiation may also be attributed to the presence of multiple populations of lineage-committed progenitor cells within adipose tissue (e.g., pre-osteoblasts, pre-myoblasts, or preadipocytes), multipotent cells from other sources (e.g., blood vessels or pericytes), or a combination of these populations. muADAS cell populations obtained from excised adipose tissue are known to contain pre-adipocytes that differentiate into mature adipocytes. The adipogenic differentiation 


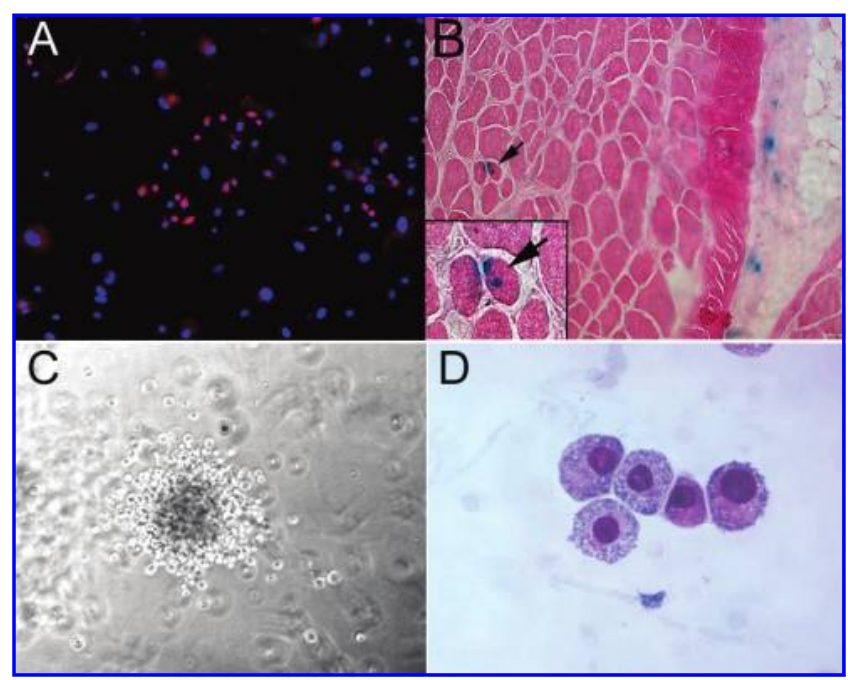

FIG. 5. (A) Murine adipose-derived adult stem (muADAS) cells cultured for 6 weeks in myogenic medium expressed desmin (red, magnification $20 \times$ ). Hoechst staining (blue) revealed the cell nuclei. (B) Few muADAS cells participated in muscle regeneration, as determined by the number of LacZ-positive myofibers in the $m d x$ mouse muscle 15 days after injection (magnification $20 \times$, bottom left-hand corner insets, $60 \times$ ). (C) Large colonies composed of small cells were found in methylcellulose cultures of muADAS cells (magnification, $5 \times$ ). (D) After picking, cytospinning, and staining the colonies with Wright-Giemsa stain, we observed granulomonocytic cells (magnification $100 \times$ )

exhibited by the muADAS cells also could be due to the commitment of existing pre-adipocytes rather than to the differentiation of multipotent cells. However, such an explanation is unconvincing in the case of humans, because as few as $0.02 \%$ of the SVF cells in adipose tissue excised from humans are pre-adipocytes that can undergo adipogenic differentiation. ${ }^{6}$ Therefore, the large-scale adipogenesis observed in our study more likely resulted from the differentiation of muADAS cells themselves.

Our observations of muADAS cells under control conditions also revealed changes in their expression of CD34 and Sca-1, both of which are progenitor cell surface markers. Our analysis of muADAS cells at 10 passages indicated that $74.94 \%$ expressed Sca- 1 and $3.25 \%$ expressed CD34. In comparison, the expression of Sca- 1 and CD34 by muADAS cells at 4 passages was $31 \%$ and $10 \%$, respectively. We are investigating whether these changes in the marker expression profile reflect the different proliferation rates of stem cells and differentiated cells. Because we isolated the cells from visceral fat, the involvement of myogenic precursor cells or satellite cells in the observed myogenic differentiation is unlikely. Furthermore, the freshly isolated muADAS cells did not express the myogenic marker desmin. Desmin was only expressed when muADAS cells were cultured in myogenic medium. This finding suggests that the myogenic differentiation observed in this study was not due to the activity of myogenic pre- cursors or proliferating myoblasts within the muADAS cell population.

The circulatory system, which contains potentially multipotent cells such as pericytes and marrow-derived mesenchymal stem cells, is another possible source of stem cell contamination of the muADAS cell population. Disruption of the blood supply during isolation could have resulted in the release of hematopoietic progenitors, which are known to give rise to hematopoietic lineages in vivo and in vitro $;{ }^{11}$ however, flow cytometry revealed no CD45-positive cells in the muADAS cell population. In addition, prior research revealed no hematopoietic cells in muADAS cell cultures at 2 to 3 passages. ${ }^{9,20}$ Although our in vitro data suggest that the muADAS cells might have at least limited hematopoietic potential, muADAS cells transplanted intravenously or intraperitoneally failed to prevent the death of lethally irradiated SJL/J mice. The immediate death of the mice after intravenous injection of muADAS cells could be due to the formation of a fat bolus in critical organs such as the brain or heart. The inability of intraperitoneally and intravenously injected muADAS cells to reconstitute the hosts' bone marrow suggests that the in vivo hematopoietic potential of these cells is, at best, limited. Because of these findings, definitive confirmation of the existence of multipotent stem cells within adipose tissue will require the isolation and characterization of multiple clones.

Researchers have demonstrated that bone marrowderived mesenchymal stem cells can differentiate toward osteogenic and chondrogenic lineages in vivo, and preliminary data suggest that these cells can facilitate the repair of bone and cartilage defects. ${ }^{21-24}$ However, harvesting bone marrow is an invasive procedure, and the marrow contains only a limited number of progenitor cells. Many studies of ADAS cells have focused on the cells' ability to differentiate toward various lineages in vitro or their potential to repair bone and cartilage in vivo; few studies have investigated the long-term fate of donor ADAS cells influenced by BMP4 in vivo. The results presented here demonstrate that ADAS cells transduced with a retrovirus containing $L a c Z$ and BMP4 genes and implanted into the skeletal muscle of mice differentiated toward the chondrogenic and osteogenic lineages and continued to survive within ectopic bone tissue 6 months after implantation. Moreover, radiographic analysis revealed that the ectopic bone formed in vivo maintained its size for 5 months (from 4 to 24 weeks after implantation, Figs. 3B, 4A). Histologic analysis also revealed a greater amount of dense bone and cartilage in the samples obtained 24 weeks after implantation than in those harvested 4 weeks after implantation. These observations indicate that the ectopic cartilage and bone tissue formed by the transduced muADAS cells was stable and persistent, which suggests that ADAS cells could serve as a good cell source for bone and cartilage regeneration.

In conclusion, these observations validate the notion that muADAS cells could serve as a cell source for gene 
therapy. The limited ability of those cells to undergo myogenic and hematopoietic differentiation restricted their utility for muscle regeneration and reconstitution of bone marrow. On the other hand, their high capacity for bone and cartilage repair and their long-term survival under the influence of BMP4 in vivo suggest that they might be a useful cell source for osteogenic and chondrogenic applications. The origin of these multipotent cells isolated from adipose tissue remains unclear and will be the focus of future research.

\section{ACKNOWLEDGMENTS}

This work was supported by a grant from the National Institutes of Health (R01 AR49684-01, R01-DE13420) and by funding from the Henry J. Mankin Endowed Chair for Orthopaedic Surgery at the University of Pittsburgh and the William F. and Jean W. Donaldson Chair at the Children's Hospital of Pittsburgh. This investigation was conducted in a facility constructed with support from Research Facilities Improvement Program Grant C06 RR-14489 from the National Center for Research Resources, National Institutes of Health. The authors thank Marcelle Huard, Jing Zhou, and Dr. Arvydas Usas for their technical contributions and Ryan Sauder for his editorial assistance with the manuscript.

\section{REFERENCES}

1. Pittenger, M.F., Mackay, A.M., Beck, S.C., Jaiswal, R.K., Douglas, R., Mosca, J.D., Moorman, M.A., Simonetti, D.W., Caraig, S., and Marshak, D.R. Multilineage potential of adult human mesenchymal stem cells. Science 284, 143, 1999.

2. Rietze, R.L., Valcanis, H., Brooker, G.F., Thomas, T., Voss, A.K., and Bartlett, P.F. Purification of a pluripotent neural stem cell from the adult mouse brain. Nature 412, 736, 2001.

3. Toma, J.G., Akhavan, M., Fernandes, K.J., Barnabe-Heider, F., Sadikot, A., Kaplan, D.R., and Miller, F.D. Isolation of multipotent adult stem cells from the dermis of mammalian skin. Nat Cell Biol 3, 778, 2001.

4. Wakitani, S., Saito, T., and Caplan, A.I. Myogenic cells derived from rat bone marrow mesenchymal stem cells exposed to 5-azacytidine. Muscle Nerve 18, 1417, 1995.

5. Qu-Petersen, Z., Deasy, B., Jankowski, J., Ikezawa, M., Cummins, J., Pruchnic, R., Mytinger, J., Cao, B., Gates, C., Wernig, A., and Huard, J. Identification of a novel population of muscle stem cells in mice: potential for muscle regeneration. J Cell Biol 157, 851, 2002.

6. Zuk, P.A., Zhu, M., Mizuno, H., Huang, J., Futrell, J.W., Katz, A.J., Benhaim, P., Lorenz, H.P., and Hedrick, M.H. Multilineage cells from human adipose tissue: implications for cell-based therapies. Tissue Eng 7, 211, 2001.

7. Zuk, P.A., Zhu, M., Ashjian, P., De Ugarte, D.A., Huang, J.I., Mizuno, H., Alfonso, Z.C., Fraser, J.K., Benhaim, P., and Hedrick, M.H. Human adipose tissue is a source of multipotent stem cells. Mol Biol Cell 13, 4279, 2002.
8. Morizono, K., De Ugarte, D.A., Zhu, M., Zuk, P., Elbarbary, A., Ashjian, P., Benhaim, P., Chen, I.S., and Hedrick, M.H. Multilineage cells from adipose tissue as gene delivery vehicles. Hum Gene Ther 14, 59, 2003.

9. Safford, K.M., Hicok, K.C., Safford, S.D., Halvorsen, Y.D., Wilkison, W.O., Gimble, J.M., and Rice, H.E. Neurogenic differentiation of murine and human adipose-derived stromal cells. Biochem Biophys Res Commun 294, 371, 2002.

10. Huang, J.I., Beanes, S.R., Zhu, M., Lorenz, H.P., Hedrick, M.H., and Benhaim, P. Rat extramedullary adipose tissue as a source of osteochondrogenic progenitor cells. Plast Reconstr Surg 109, 1033, 2002.

11. Cousin, B., Andre, M., Arnaud, E., Penicaud, L., and Casteilla, L. Reconstitution of lethally irradiated mice by cells isolated from adipose tissue. Biochem Biophys Res Commun 301, 1016, 2003.

12. Tholpady, S.S., Katz, A.J., and Ogle, R.C. Mesenchymal stem cells from rat visceral fat exhibit multipotential differentiation in vitro. Anat Rec 272, 398, 2003.

13. Johnstone, B., Hering, T.M., Caplan, A.I., Goldberg, V.M., and Yoo, J.U. In vitro chondrogenesis of bone marrowderived mesenchymal progenitor cells. Exp Cell Res 238, 265, 1998.

14. Mackay, A.M., Beck, S.C., Murphy, J.M., Barry, F.P., Chichester, C.O., Pittenger, M.F. Chondrogenic differentiation of cultured human mesenchymal stem cells from marrow. Tissue Eng 4, 415, 1998.

15. Barry, F., Boynton, R.E., Liu, B., and Murphy, J.M. Chondrogenic differentiation of mesenchymal stem cells from bone marrow: differentiation-dependent gene expression of matrix components. Exp Cell Res 268, 189, 2001.

16. Lee, J.Y., Qu-Petersen, Z., Cao, B., Kimura, S., Jankowski, R., Cummins, J., Usas, A., Gates, C., Robbins, P., Wernig, A., and Huard, J. Clonal isolation of muscle-derived cells capable of enhancing muscle regeneration and bone healing. J Cell Biol 150, 1085, 2000.

17. Peng, H., Chen, S.T., Wergedal, J.E., Polo, J.M., Yee, J.K., Lau, K.H., and Baylink, D.J. Development of an MFG-based retroviral vector system for secretion of high levels of functionally active human BMP4. Mol Ther 4, 95, 2001.

18. Partridge, T.A., Morgan, J.E., Coulton, G.R., Hoffman, E.P., and Kunkel, L.M. Conversion of mdx myofibers from dystrophin negative to positive by injection of normal myoblasts. Nature 337, 176, 1989.

19. Cao B.H., Zheng B., Jankowski R.J., Kimura S., Ikezawa M., Deasy B., Cummins J., Epperly M., Qu-Petersen Z., and Huard J. Muscle stem cells differentiate into hematopoietic lineage but retain myogenic potential. Nature Cell Biol $\mathbf{5}$, 640, 2003.

20. Gronths, S., Franklin, D.M., Leddy, H.A., Robey, P.G., Storms, R.W., and Gimble, J.M. Surface protein characterization of human adipose tissue-derived stromal cells. J Cell Physiol 189, 54, 2001.

21. Krebsbach, P.H., Kuznetsov, S.A., Satomura, K., Emmons, R.V., Rowe, D.W., and Robey, P.G. Bone formation in vivo: comparison of osteogenesis by transplanted mouse and human marrow stromal fibroblasts. Transplantation 63, 1059, 1997.

22. Krebsbach, P.H., Mankani, M.H., Satomura, K., Kuznetsov, S.A., and Robey, P.G. Repair of craniotomy defects using bone marrow stromal cells. Transplantation 66, 1272, 1998. 
23. Mendes, S.C., Tibbe, J.M., Veenhof, M., Bakker, K., Both, S., Platenburg, P.P., Oner, F.C., de Bruijn, J.D., and van Blitterswijk, C.A. Bone tissue-engineered implants using human bone marrow stromal cells: effect of culture conditions and donor age. Tissue Eng 8, 911, 2002.

24. Mendes, S.C., Tibbe, J.M., Veenhof, M., Both, S., Oner, F.C., van Blitterswijk, C.A., and de Bruijn, J.D. Relation between in vitro and in vivo osteogenic potential of cultured human bone marrow stromal cells. J Mater Sci Mater Med 15, 1123, 2004.
Address reprint requests to: Johnny Huard, Ph.D. 4100 Rangos Research Center, Children's Hospital of Pittsburgh 3460 Fifth Avenue Pittsburgh, PA 15213

E-mail: jhuard@pitt.edu 



\section{This article has been cited by:}

1. Jing-Jing Sun, Xiao-Hui Zheng, Li-Ya Wang, Lei Liu, Wei Jing, Yun-Feng Lin, Weidong Tian, Wei Tang, Jie Long. 2014. New bone formation enhanced by ADSCs overexpressing hRunx2 during mandibular distraction osteogenesis in osteoporotic rabbits. Journal of Orthopaedic Research 32:5, 709-720. [CrossRef]

2. Haixiang Liang, Xudong Li, Adam L. Shimer, Gary Balian, Francis H. Shen. 2014. A novel strategy of spine defect repair with a degradable bioactive scaffold preloaded with adipose-derived stromal cells. The Spine Journal 14:3, 445-454. [CrossRef]

3. Gong Lunli, Zhou Xiao, Wu Yaohao, Zhang Yun, Wang Chen, Zhou Heng, Guo Fangfang, Cui Lei. 2014. Proteomic Analysis Profile of Engineered Articular Cartilage with Chondrogenic Differentiated Adipose Tissue-Derived Stem Cells Loaded Polyglycolic Acid Mesh for Weight-Bearing Area Defect Repair. Tissue Engineering Part A 20:3-4, 575-587. [Abstract] [Full Text HTML] [Full Text PDF] [Full Text PDF with Links] [Supplemental Material]

4. Keum Sil Lee, Hye Won Kang, Hoon Taek Lee, Hye-Jin Kim, Chan-Lan Kim, Jae-Young Song, Kyung Woo Lee, Sang-Ho Cha. 2013. Sequential sub-passage decreases the differentiation potential of canine adipose-derived mesenchymal stem cells. Research in Veterinary Science. [CrossRef]

5. Qi Li, Jianliu Wang, Haifeng Liu, Bing Xie, Lihui Wei. 2013. Tissue-engineered mesh for pelvic floor reconstruction fabricated from silk fibroin scaffold with adipose-derived mesenchymal stem cells. Cell and Tissue Research 354:2, 471-480. [CrossRef]

6. Ryan Berry, Elise Jeffery, Matthew S. Rodeheffer. 2013. Weighing in on Adipocyte Precursors. Cell Metabolism . [CrossRef]

7. Chao Wei, Xing Liu, Jia Tao, Ronghua Wu, Pengfei Zhang, Yani Bian, Yunsheng Li, Fugui Fang, Yunhai Zhang. 2013. Effects of vitamin $\mathrm{C}$ on characteristics retaining of in vitro-cultured mouse adipose-derived stem cells. In Vitro Cellular \& Developmental Biology - Animal . [CrossRef]

8. Bo Zheng, Guangheng Li, William C.W. Chen, Bridget M. Deasy, Jonathan B. Pollett, Bin Sun, Lauren Drowley, Burhan Gharaibeh, Arvydas Usas, Bruno Péault, Johnny Huard. 2013. Human myogenic endothelial cells exhibit chondrogenic and osteogenic potentials at the clonal level. Journal of Orthopaedic Research 31:7, 1089-1095. [CrossRef]

9. K.S. Lee, S.H. Cha, H.W. Kang, J.Y. Song, K.W. Lee, K.B. Ko, H.T. Lee. 2013. Effects of Serial Passage on the Characteristics and Chondrogenic Differentiation of Canine Umbilical Cord Matrix Derived Mesenchymal Stem Cells. Asian-Australasian Journal of Animal Sciences 26:4, 588-595. [CrossRef]

10. Jingang Xiao, Xiaojuan Yang, Wei Jing, Weihua Guo, Qince Sun, Yunfeng Lin, Lei Liu, Wentong Meng, Weidong Tian. 2013. Adipogenic and osteogenic differentiation of Lin-CD271+Sca-1+ adipose-derived stem cells. Molecular and Cellular Biochemistry . [CrossRef]

11. Shijia Zhang, Svitlana D Danchuk, Kathleen MP Imhof, Julie A Semon, Brittni A Scruggs, Ryan W Bonvillain, Amy L Strong, Jeffrey M Gimble, Aline M Betancourt, Deborah E Sullivan, Bruce A Bunnell. 2013. Comparison of the therapeutic effects of human and mouse adipose-derived stem cells in a murine model of lipopolysaccharide-induced acute lung injury. Stem Cell Research \& Therapy 4:1, 13. [CrossRef]

12. Minjung Song, Mitra Lavasani, Seth D Thompson, Aiping Lu, Bahar Ahani, Johnny Huard. 2013. Muscle-derived stem/ progenitor cell dysfunction in Zmpste24-deficient progeroid mice limits muscle regeneration. Stem Cell Research \& Therapy 4:2, 33. [CrossRef]

13. Patricia Zuk. 2013. Adipose-Derived Stem Cells in Tissue Regeneration: A Review. ISRN Stem Cells 2013, 1-35. [CrossRef]

14. Jong Woo Choi, Eun Jung Park,, Heung Soo Shin, Il Seob Shin, Jung Chan Ra, Kyung Suk Koh. 2012. In Vivo Differentiation of Undifferentiated Human Adipose Tissue-Derived Mesenchymal Stem Cells in Critical-Sized Calvarial Bone Defects. Annals of Plastic Surgery 1. [CrossRef]

15. Yaling Shi, Jerry R. Niedzinski, Adrian Samaniego, Simon Bogdansky, Brent L. Atkinson. 2012. Adipose-Derived Stem Cells Combined with a Demineralized Cancellous Bone Substrate for Bone Regeneration. Tissue Engineering Part A 18:13-14, 1313-1321. [Abstract] [Full Text HTML] [Full Text PDF] [Full Text PDF with Links]

16. William P. Cawthorn, Erica L. Scheller, Ormond A. MacDougald. 2012. Adipose tissue stem cells: the great WAT hope. Trends in Endocrinology \& Metabolism 23:6, 270-277. [CrossRef]

17. Tomoyuki Matsumoto, Sheila M. Ingham, Yutaka Mifune, Aki Osawa, Alison Logar, Arvydas Usas, Ryosuke Kuroda, Masahiro Kurosaka, Freddie H. Fu, Johnny Huard. 2012. Isolation and Characterization of Human Anterior Cruciate Ligament-Derived Vascular Stem Cells. Stem Cells and Development 21:6, 859-872. [Abstract] [Full Text HTML] [Full Text PDF] [Full Text PDF with Links]

18. Yimu Zhao, Stephen D. Waldman, Lauren E. Flynn. 2012. The Effect of Serial Passaging on the Proliferation and Differentiation of Bovine Adipose-Derived Stem Cells. Cells Tissues Organs 195:5, 414-427. [CrossRef] 
19. Eileen M. Shore. 2012. Fibrodysplasia ossificans progressiva: a human genetic disorder of extraskeletal bone formation, or-how does one tissue become another?. Wiley Interdisciplinary Reviews: Developmental Biology 1:1, 153-165. [CrossRef]

20. Hong-ming Du, Xiao-hui Zheng, Li-ya Wang, Wei Tang, Lei Liu, Wei Jing, Yun-feng Lin, Wei-dong Tian, Jie Long. 2012. The Osteogenic Response of Undifferentiated Human Adipose-Derived Stem Cells under Mechanical Stimulation. Cells Tissues Organs 196:4, 313-324. [CrossRef]

21. Xiaodong Mu, Guosheng Xiang, Christopher R. Rathbone, Haiying Pan, Ian H. Bellayr, Thomas J. Walters, Yong Li. 2011. Slow-Adhering Stem Cells Derived from Injured Skeletal Muscle Have Improved Regenerative Capacity. The American Journal of Pathology 179:2, 931-941. [CrossRef]

22. F. Hildner, C. Albrecht, C. Gabriel, H. Redl, M. van Griensven. 2011. State of the art and future perspectives of articular cartilage regeneration: a focus on adipose-derived stem cells and platelet-derived products. Journal of Tissue Engineering and Regenerative Medicine 5:4, e36-e51. [CrossRef]

23. József Gábor Joó. 2011. Magzatvíz-eredetű őssejtek: a regeneratív medicina legújabb lehetőségei. Orvosi Hetilap 152:15, 581-587. [CrossRef]

24. Xiaodong Mu, Yong Li. 2011. Conditional TGF- $\beta 1$ treatment increases stem cell-like cell population in myoblasts. Journal of Cellular and Molecular Medicine 15:3, 679-690. [CrossRef]

25. Akon Higuchi, Chung-Wei Chuang, Qing-Dong Ling, See-Chang Huang, Ling-Mei Wang, Hui Chen, Yung Chang, HanChow Wang, Jun-Tang Bing, Yu Chang. 2011. Differentiation ability of adipose-derived stem cells separated from adipose tissue by a membrane filtration method. Journal of Membrane Science 366:1-2, 286-294. [CrossRef]

26. M. G. Valorani, A. Germani, W. R. Otto, L. Harper, A. Biddle, C. P. Khoo, W. R. Lin, M. I. Hawa, P. Tropel, M. P. Patrizi, P. Pozzilli, M. R. Alison. 2010. Hypoxia increases Sca-1/CD44 co-expression in murine mesenchymal stem cells and enhances their adipogenic differentiation potential. Cell and Tissue Research 341:1, 111-120. [CrossRef]

27. Jeffrey M. Gimble, Bruce A. Bunnell, Farshid GuilakBiology of Adipose Tissue Stem Cells 69-80. [CrossRef]

28. Hirotaka Suga, Daisuke Matsumoto, Hitomi Eto, Keita Inoue, Noriyuki Aoi, Harunosuke Kato, Jun Araki, Kotaro Yoshimura. 2009. Functional Implications of CD34 Expression in Human Adipose-Derived Stem/Progenitor Cells. Stem Cells and Development 18:8, 1201-1210. [Abstract] [Full Text PDF] [Full Text PDF with Links] [Supplemental Material]

29. Lei Cui, Yaohao Wu, Lian Cen, Heng Zhou, Shuo Yin, Guangpeng Liu, Wei Liu, Yilin Cao. 2009. Repair of articular cartilage defect in non-weight bearing areas using adipose derived stem cells loaded polyglycolic acid mesh. Biomaterials 30:14, 2683-2693. [CrossRef]

30. Samyra El-ftesi, Edward I. Chang, Michael T. Longaker, Geoffrey C. Gurtner. 2009. Aging and Diabetes Impair the Neovascular Potential of Adipose-Derived Stromal Cells. Plastic and Reconstructive Surgery 123:2, 475-485. [CrossRef]

31. Hariharan Thangarajah, Ivan N. Vial, Edwin Chang, Samyra El-Ftesi, Michael Januszyk, Edward I. Chang, Josemaria Paterno, Evgenios Neofytou, Michael T. Longaker, Geoffrey C. Gurtner. 2009. IFATS Collection: Adipose Stromal Cells Adopt a Proangiogenic Phenotype Under the Influence of Hypoxia. Stem Cells 27:1, 266-274. [CrossRef]

32. Tomoyuki Matsumoto, Seiji Kubo, Laura B. Meszaros, Karin A. Corsi, Gregory M. Cooper, Guangheng Li, Arvydas Usas, Aki Osawa, Freddie H. Fu, Johnny Huard. 2008. The influence of sex on the chondrogenic potential of muscle-derived stem cells: Implications for cartilage regeneration and repair. Artbritis \& Rheumatism 58:12, 3809-3819. [CrossRef]

33. Wang Shu, Yang Ting Shu, Han Bao Shi, Liu Xiao Fei, Wang Lei, Qi Guan ming. 2008. An easy method to discover cell membrane antigen with atomic force microscopy. Molecular Biology Reports 35:4, 557-561. [CrossRef]

34. Matthew S. Rodeheffer, Kıvanç Birsoy, Jeffrey M. Friedman. 2008. Identification of White Adipocyte Progenitor Cells In Vivo. Cell 135:2, 240-249. [CrossRef]

35. Daniel J. Weiss. 2008. Stem cells and cell therapies for cystic fibrosis and other lung diseases. Pulmonary Pharmacology \& Therapeutics 21:4, 588-594. [CrossRef]

36. Wouter J. F. M. Jurgens, Maikel J. Oedayrajsingh-Varma, Marco N. Helder, Behrouz ZandiehDoulabi, Tabitha E. Schouten, Dirk J. Kuik, Marco J. P. F. Ritt, Florine J. Milligen. 2008. Effect of tissue-harvesting site on yield of stem cells derived from adipose tissue: implications for cell-based therapies. Cell and Tissue Research 332:3, 415-426. [CrossRef]

37. PATRICIA A. ZUK. 2008. Tissue Engineering Craniofacial Defects With Adult Stem Cells? Are We Ready Yet?. Pediatric Research 63:5, 478-486. [CrossRef]

38. Feng Lu, Hiroshi Mizuno, Cagri A. Uysal, Xiaobo Cai, Rei Ogawa, Hiko Hyakusoku. 2008. Improved Viability of Random Pattern Skin Flaps through the Use of Adipose-Derived Stem Cells. Plastic and Reconstructive Surgery 121:1, 50-58. [CrossRef]

39. Jeffrey M. Gimble, Farshid Guilak, Mark E. Nuttall, Solomon Sathishkumar, Martin Vidal, Bruce A. Bunnell. 2008. In vitro Differentiation Potential of Mesenchymal Stem Cells. Transfusion Medicine and Hemotherapy 35:3, 228-238. [CrossRef] 
40. Yash M. Kolambkar, Alexandra Peister, Shay Soker, Anthony Atala, Robert E. Guldberg. 2007. Chondrogenic differentiation of amniotic fluid-derived stem cells. Journal of Molecular Histology 38:5, 405-413. [CrossRef]

41. Bo Zheng, Baohong Cao, Mihaela Crisan, Bin Sun, Guangheng Li, Alison Logar, Solomon Yap, Jonathan B Pollett, Lauren Drowley, Theresa Cassino, Burhan Gharaibeh, Bridget M Deasy, Johnny Huard, Bruno Péault. 2007. Prospective identification of myogenic endothelial cells in human skeletal muscle. Nature Biotechnology 25:9, 1025-1034. [CrossRef]

42. Barbara Gawronska-Kozak, Jessica A. Manuel, Veronica Prpic. 2007. Ear mesenchymal stem cells (EMSC) can differentiate into spontaneously contracting muscle cells. Journal of Cellular Biochemistry 102:1, 122-135. [CrossRef]

43. Irene Román, Marta Vilalta, Julio Rodriguez, Annette M. Matthies, Samer Srouji, Erella Livne, Jeffrey A. Hubbell, Nuria Rubio, Jerónimo Blanco. 2007. Analysis of progenitor cell-scaffold combinations by in vivo non-invasive photonic imaging. Biomaterials 28:17, 2718-2728. [CrossRef]

44. Guangwu Xu, Liying Zhang, Guangwen Ren, Zengrong Yuan, Yingyu Zhang, Robert C Zhao, Yufang Shi. 2007. Immunosuppressive properties of cloned bone marrow mesenchymal stem cells. Cell Research . [CrossRef] 